\title{
Practical bit error rate measurements on fibre optic communications links in student teaching laboratories
}

Douglas Walsh, David Moodie, lain Mauchline, Steve Conner, Walter Johnstone, et al.

Douglas Walsh, David Moodie, lain Mauchline, Steve Conner, Walter Johnstone, Brian Culshaw, "Practical bit error rate measurements on fibre optic communications links in student teaching laboratories," Proc. SPIE 9664, Ninth International Topical Meeting on Education and Training in Optics and Photonics, 96642I (24 October 2005); doi: 10.1117/12.2207674 
Ref ETOP021

\title{
Practical Bit Error Rate Measurements on Fibre Optic Communications Links in Student Teaching Laboratories
}

\author{
Douglas Walsh ${ }^{1}$, David Moodie ${ }^{1}$, lain Mauchline ${ }^{1}$, Steve Conner ${ }^{1}$, Walter Johnstone ${ }^{2}$, \\ Brian Culshaw ${ }^{2}$
}

${ }^{1}$ OptoSci Ltd, 141 St. James Rd., Glasgow, G4 OLT, Scotland, UK www.optosci.com

T: +44 141552 7020, F: +44 141552 3886, E: info@optosci.com

${ }^{2}$ EEE Dept., University of Strathclyde, 204 George St., Glasgow, G1 1XW Scotland, UK

\begin{abstract}
In this paper we describe the principles and design of a fibre optic communications teaching package and a cost effective extension module to this kit which enables students to investigate the effects of noise, attenuation and dispersion on the bit error rate at the receiver of laser and LED based digital fibre optic communication systems.
\end{abstract}

\section{Keywords}

photonics teaching laboratories, educational experiments, photonics education, optical communications, bit error rates, eye diagrams, BER.

\section{Summary}

Optical fibre communications has proved to be one of the key application areas, which created, and ultimately propelled the global growth of the photonics industry over the last twenty years. Consequently the teaching of the principles of optical fibre communications has become integral to many university courses covering photonics technology. However to reinforce the fundamental principles and key technical issues students examine in their lecture courses and to develop their experimental skills, it is critical that the students also obtain hands-on practical experience of photonics components, instruments and systems in an associated teaching laboratory. In recognition of this need OptoSci Ltd, in collaboration with academics at Strathclyde and Heriot-Watt Universities, has commercially developed a suite of fully self-contained laboratory based photonics teaching packages for use in universities, colleges, and industrial training centres.

One of these teaching packages covers experiments in fibre optic communications (EDCOM). ED-COM enables students to; investigate the characteristics of the individual communications system components (sources, transmitters, fibre, receiver), examine and interpret the overall system performance limitations imposed by attenuation and dispersion, conduct system design and performance analysis. To further enhance the experimental programme examined in the fibre optic communications kit, an extension module to ED-COM has recently been introduced examining one of the most significant performance parameters of digital communications systems, the bit error rate (BER). This add-on module, BER(COM), enables students to generate, evaluate and investigate signal quality trends by examining eye patterns, and explore the bit-rate limitations imposed on communication systems by noise, attenuation and dispersion. 
This paper will examine the educational objectives, background theory, and typical results for these educator kits, with particular emphasis on BER(COM).

\section{Introduction}

In Engineering and Physics courses there is no substitute for student hands-on practical experience of measurements made using real hardware and signals. Cost issues and simplicity often drive the busy tutor to using computer simulations and until now this has been the standard approach to student learning in the area of signal recovery and bit error rate analysis in digital optical communications systems. Here we describe the principles and design of a fibre optic communications teaching package and a cost effective extension module to this kit which enables students to investigate the effects of noise, attenuation and dispersion on the bit error rate at the receiver of laser and LED based digital fibre optic communication systems. 


\section{Design Philosophy}

The overall educational aims of a teaching laboratory are to enable students to consolidate their understanding and knowledge of photonics as presented in an accompanying lecture course and to acquire practical experience of the design, analysis and characteristics of photonics components and systems. To achieve these aims it is essential to take a fully integrated approach to the design of laboratory based photonics teaching packages including the design of dedicated hardware, experimental procedures, exercises and manuals. To ensure that all desirable educational objectives are met and that all of the most important scientific and technical principles, issues and phenomena are addressed, we have developed our suite of fully integrated laboratory based teaching packages in accordance with the following design rules

- Define the educational objectives in terms of the physical principles, important technical features, design issues and performance characteristics which must be addressed, with particular attention to facilitating student understanding and ability to implement concepts.

- Define the experiments to meet these performance objectives.

- Design the dedicated (custom) hardware to enable the proposed experimental investigation whilst keeping costs within realistic academic teaching budgets.

- Formulate the experimental procedure and manuals to guide the students through the investigation and results analysis (in some cases more open ended investigations may be formulated with minimal guidance to the students).

- Formulate tutorial exercises and case studies to relate the results to real world devices and systems.

The primary constraint is cost and the final packages must be affordable within higher education budgets. In general, the packages have been designed as far as possible to be self-contained so that as little ancillary equipment as possible is required. However, where it is advantageous and cost effective to use equipment normally available in student laboratories, the packages have been designed to be compatible with the capabilities of such equipment e.g. a $20 \mathrm{MHz}$ or $50 \mathrm{MHz}$ oscilloscope.

Using the design principles referred to above, OptoSci, in collaboration with academics at the University of Strathclyde, has commercially developed a unique range of fully selfcontained, laboratory based teaching packages for use in universities, colleges, and industrial training centres. The current series of educator kits allow students to perform detailed experimental investigations in key areas of optics, optoelectronics and optical fibre communications ${ }^{1}$.

\section{ED-COM Fibre Optic Communications}

Optical fibre information transmission links enable more information to be transmitted over greater distance than any other communications technology. Hence, they have all but completely replaced copper based systems as the primary choice for global and local telecommunications systems. Consequently, a working knowledge and understanding of the components of a fibre optic communications system and the limits imposed on the system performance by the component characteristics are invaluable for any student intending to work with photonics systems. To satisfy this requirement OptoSci introduced ED-COM, its Fibre Optic Communications educator kit in 1995 (see 
Figure 1). The objectives of the ED-COM experiments are to enable students to experimentally explore, investigate and interpret:

- the main characteristics of the major components of a fibre optic communications system i.e. the source / transmitter, the fibre channel (attenuation, dispersion, pulse spreading etc.) and the receiver

- the overall system performance limitations imposed by the component characteristics

- $\quad$ the maximum possible link length limited by attenuation

- the bit rate (and bandwidth) / length products determined by fibre dispersion

- optical communication system design and performance analysis.

To achieve these objectives the students carry out the following investigations:

\section{Stage 1. Power Budgets}

- Measurement of the power / current characteristics, bias points and launched powers of the laser and LED transmitters.

- Measurement of connector losses.

- Measurement of the fibre attenuation coefficient.

- Determination of fibre link lengths

- Measurement of the receiver noise and sensitivity.

- Calculation and comparison of the attenuation limited link lengths for the laser and LED transmitters.

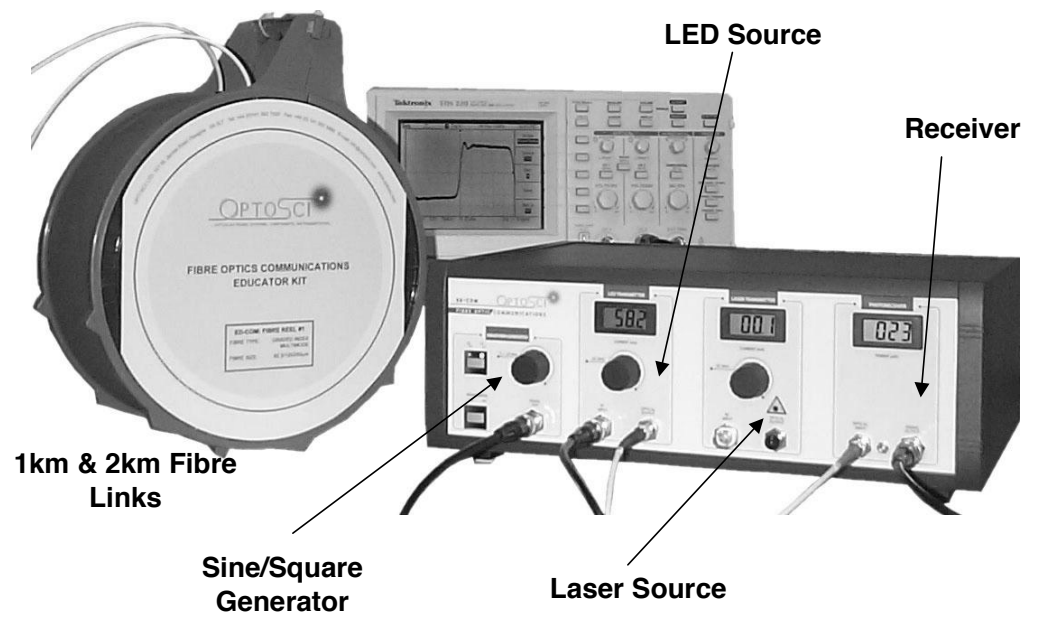

Figure 1: ED-COM Fibre Optic Communications educator kit 
Stage 2. Temporal Characteristics

- Measurement of the step function response of the transmitter / receiver, the system and the fibre using both the laser and the LED. This enables the determination of

- the fibre impulse response for both the laser and the LED

- the bit rate distance products for both the laser and LED transmitters.

- Measurement of the analogue signal frequency response of the transmitter / receiver, the system and the fibre, leading to determination of

- the analogue bandwidth and bandwidth.distance products of the fibre for both the LED and laser sources. It is interesting to compare the directly measured bandwidth with that obtained from the step response.

- Determination of the fibre intermodal and material dispersion coefficients.

Stage 3. System Performance and Analysis

- The design of systems to meet a given specification using the measured data.

- Analysis of the performance of systems to determine if they will meet a required specification.

- Design and performance analysis for state of the art systems at $1.3 \& 1.55 \mu \mathrm{m}$ to compare with the results for the system investigated.

The background theory and sample results for ED-COM will not be expounded here as they have been reported in detail previously ${ }^{2,3}$.

\section{BER(COM) Bit Error Rate in Optical Communications \\ Introduction}

The bit error rate (BER) is the most significant performance parameter of any digital communications system. It is a measure of the probability that any given bit will have been received in error. For example a standard maximum bit error rate specified for many systems is $10^{-9}$. This means that the receiver is allowed to generate a maximum of 1 error in every $10^{9}$ bits of information transmitted or, putting it another way, the probability that any received bit is in error is $10^{-9}$.

The BER depends primarily on the signal to noise ratio (SNR) of the received signal which in turn is determined by the transmitted signal power, the attenuation of the link, the link dispersion and the receiver noise. Measurement of the BER is not a trivial process and requires sophisticated and expensive equipment to achieve accuracy, particularly at high bit rates. However, the effects of noise and other signal degradation processes can be investigated qualitatively and perhaps even in a pseudo quantitative manner by generating the "eye pattern" or "eye diagram" for the system. Certainly signal and BER degradation trends can be readily seen due to the effects of signal attenuation and dispersion.

Recently OptoSci has introduced an extension module to its very popular ED-COM Fibre Optic Communications educator kit examining eye diagrams and bit error rates. This new module is called BER(COM) and the key educational objectives for the students during the experimental investigation in this module are 
- to learn how to generate and evaluate eye diagrams for a transmitted pseudo random bit stream (PRBS)

- to investigate the trends in signal quality by evaluating the eye diagrams for the many permutations of system design allowed by ED-COM, and

- to gain an appreciation of the effects of noise, attenuation and dispersion on the eye diagrams and the BER

$\mathrm{BER}(\mathrm{COM})$ is an extension module to the ED-COM educator kit and must be used in conjunction with this kit (see Figure 2). BER(COM) consists of a PRBS generator with data and clock output ports. The non return to zero PRBS pattern repeats every 511 $\left(2^{\mathrm{N}=9}-1\right)$ bits and in random order all possible bit sequences for $\mathrm{N}$ bit words up to $\mathrm{N}=9$. The PRBS bit rate may be varied from $1 \mathrm{Mbit} / \mathrm{s}$ to $40 \mathrm{Mbit} / \mathrm{s}$ using the rotary control knob on the front fascia and the bit rate is displayed on the front panel LCD. When used with the ED-COM fibre reels, the additional $2 \mathrm{~km}$ graded index fibre reel supplied allows fibre links of $1 \mathrm{~km}$ to $5 \mathrm{~km}$ to be investigated with the ED-COM laser, LED and receiver.

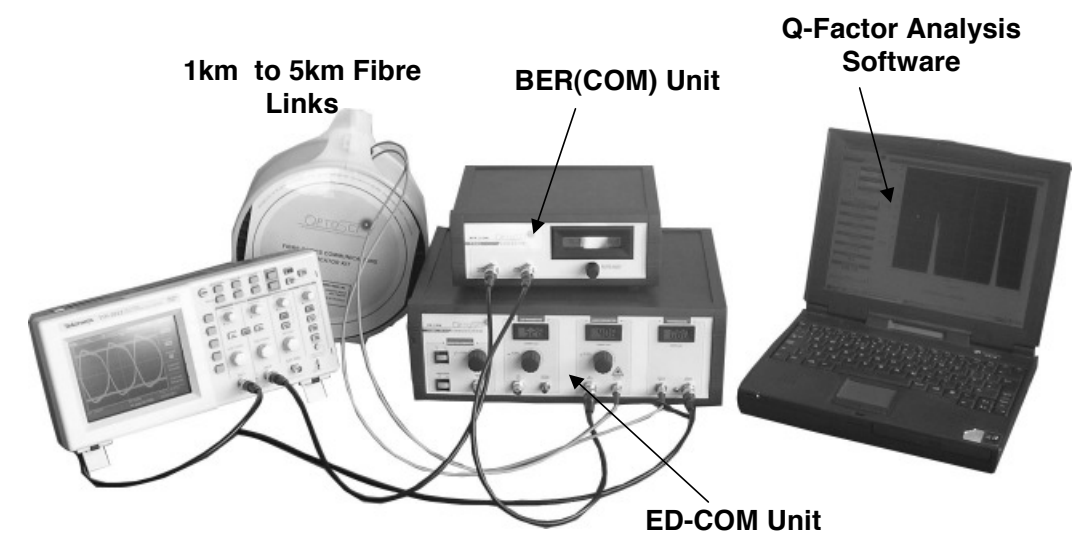

Figure 2: ED-COM and BER(COM) units in operation

Q-factor Analysis software (Figure 3 ) is also provided with BER(COM) to enable a digital oscilloscope to sample the received signals in the centre of the bit period, transfer the samples to a PC and then analyse them. The analysis algorithms in the software enable the construction of a signal level histogram (i.e. a plot of the number of samples occurring in a narrow voltage range versus voltage) which is essentially the probability distribution of the signal levels around the mean 0 and 1 levels. Theoretical Gaussian distributions are curve fitted within the software to the measured distributions, the signal level (noise) variances are extracted and the Q-factors and BER are determined. As with all of OptoSci's educational kits, a detailed student manual covering the relevant background theory and experimental procedure and a comprehensive instructor manual with full sample results for all the experiments are also provided. 


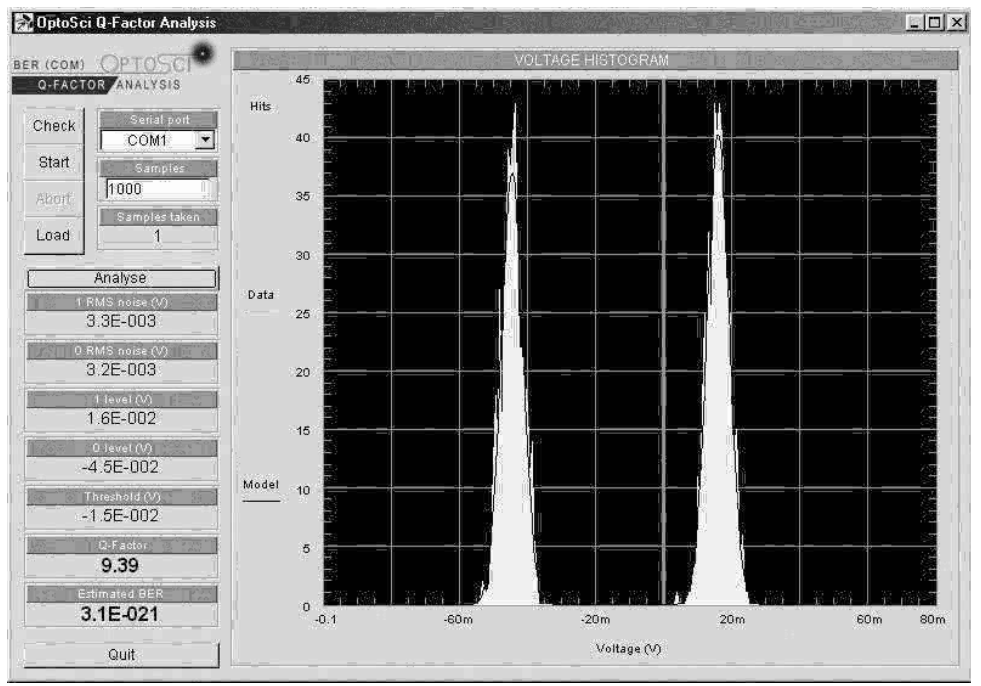

Figure 3: Q-factor analysis software showing voltage histogram and analysis results

\section{Theory}

In a digital optical telecommunications receiver, the incident signals are sampled in the centre of the bit period and the sampled level is compared to a threshold to determine the presence of a one or zero. With threshold detection of this nature errors arise when noise in the system pulls a one signal level below threshold at the sampling point and pushes a zero above threshold (see Figure 4).

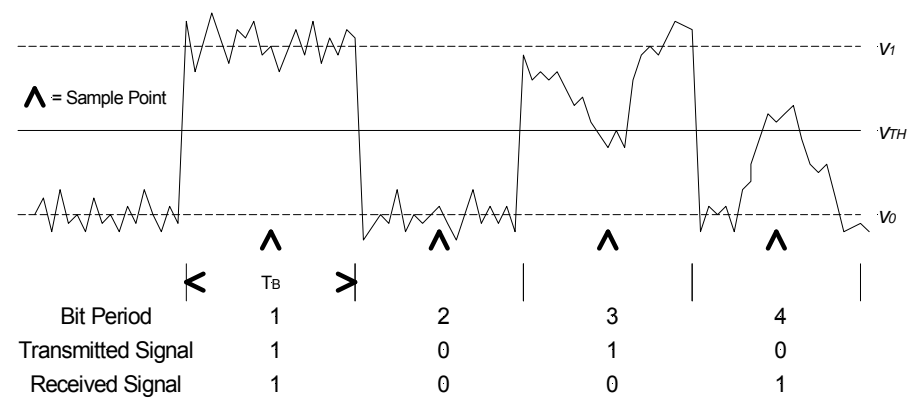

Figure 4.: Threshold detection in digital receivers, illustrating the possibility of errors in the presence of random noise

Clearly, due to the random nature of the noise the total signal can be above threshold for a 0 and below for a 1 giving rise to a non zero probability of errors occurring with an associated bit error rate (BER) defined by:-

$$
B E R=\frac{N_{e}}{N_{t}}
$$


where $N_{e}$ is the number of errors occurring in time $t$ and $N_{t}$ is the number of bits arriving in time $t$. The bit error rate (BER) is simply the probability that an error will occur in a given bit period.

Clearly from Figure 4, if we have greater SNRs, we can set higher threshold levels $\left(v_{\text {th }}\right.$ in Figure 4) relative to the noise and hence reduce the probability of error. It is standard practice in digital telecommunications to ensure by design that a specified BER is achieved. For some applications the maximum specified BER is $10^{-9}$ implying that only one error in $10^{9}$ received bits is tolerated e.g. 1 error per second at a bit rate of 1Gbit/s. For telecommunications applications the specified maximum BER falls in the range $10^{-9}$ to $10^{-12}$.

To calculate the probability of error, we need to establish the noise statistics and compute the probability that the noise level at any given sampling point pushes the signal to the wrong side of the threshold for a 1 or 0 transmitted. Figure 5 illustrates the basics of the calculation. Essentially, at the sampling point in any bit period, the signal is varying randomly about some mean value due to the random noise fluctuations i.e. the sampled signal level is a random statistical variable about its mean. To determine the probability of error we need to know the signal probability distribution functions $\left[p_{i}(v) d v, i=0\right.$ or 1$]$ for the 0 and 1 levels i.e. the probability that the sampled signal level will fall between level $v$ and $v$ $+d v$ as a function of $v$ for a received 0 and 1 signal, where $v_{0}$ and $v_{1}$ are the mean levels for a received 0 and 1 respectively. Illustrations of such functions are presented in Figure 5 . It should be noted that the probability distribution functions (PDFs) for 0 and 1 received signals may be different since different noise processes may dominate the two regimes. This is reflected in Figure 5. However, for many applications, particularly when thermal noise limits apply, the PDFs are identical for both 0 and 1 levels.

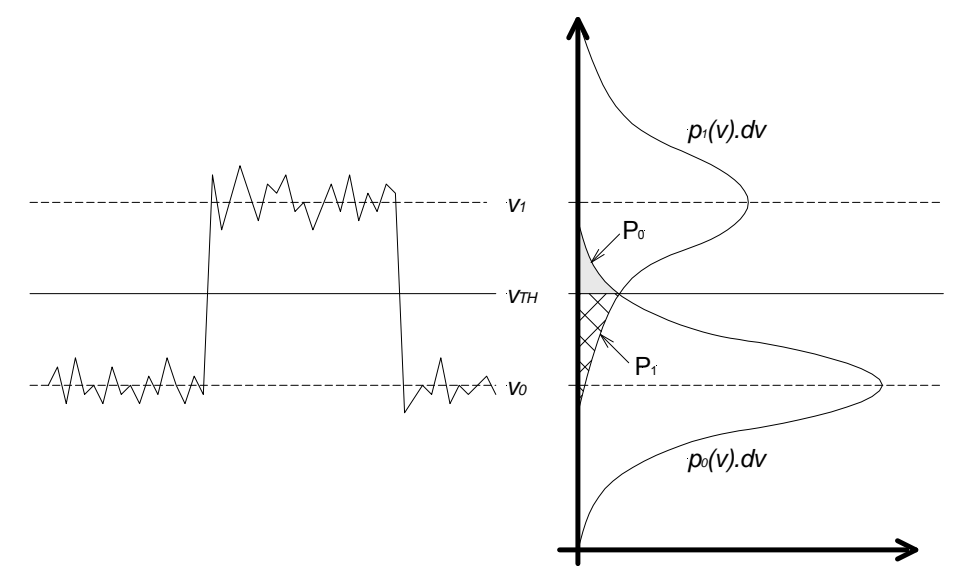

Figure 5: PDFs $\left[p_{i}(v) d v_{i}, i=0,1\right]$ for levels of 0 and $1\left(v_{0}\right.$ and $\left.v_{1}\right)$ in the presence of random (Gaussian) noise. The probabilities of error on a 0 signal $\left(\mathrm{P}_{0}\right.$-shaded) and 1 signal $\left(\mathrm{P}_{1}\right.$-hatched) are also indicated

The total probability that a 0 signal is recorded in error as 1 is the area under the probability distribution function, $p_{0}(v) . d v$, for $v>v_{t h}$ (shaded area of Figure ). Also, the total probability that a 1 signal is recorded in error as 0 is the area under the probability distribution function, 
$p_{1}(v) . d v$, for $v<v_{\text {th }}$ (hatched area of Figure ). Hence, the probabilities $\left(P_{i}\right)$ that a received signal of level $i$ is recorded wrongly for $i=0$ and $i=1$ respectively are:

$$
P_{0}=\int_{v_{t h}}^{\infty} p_{0} d v \text { and } P_{1}=\int_{-\infty}^{v_{t h}} p_{1} d v
$$

In the interests of simplicity, we shall adopt a useful approximation in assuming that the noise is a Gaussian random variable. This means that the PDFs $\left[p_{i}(v) d v, i=0\right.$ or 1$]$ for the sampled signal voltage at the receiver output are Gaussian functions with a mean voltage of $v_{i}$ and a variance of $\sigma_{i}^{2}=v_{N}^{2}$, where $v_{N}$ is the root mean square (rms) noise voltage. Assuming equal numbers of $1 \mathrm{~s}$ and $0 \mathrm{~s}$ in the bit stream and evaluating the integrals of equations (2) and (3), the BER, or error probability, $P_{e}$ can now be written $\mathrm{as}^{4}$

$$
B E R=P_{e}=\frac{1}{2}[1-\operatorname{erf}(Q / \sqrt{2})]
$$

where, for thermal noise limited detection (equal noise on 0 and 1 levels)

$$
Q=\left(v_{1}-v_{\text {th }}\right) / v_{N} \text { and } v_{\text {th }}=\left(v_{1}-v_{0}\right) / 2 \text {. }
$$

For this parameter set, $\mathrm{Q}$ is in fact the signal to noise ratio and the threshold voltage is half of the received average 1 level. Hence we can easily determine the BER from equation (4).

Figure 6 shows the variation in the BER as a function of $Q$ according to equation 4 . The maximum acceptable bit error rate in a telecommunications system is $10^{-9}$ and system specifications often demand BERs down to $10^{-12}$. Such specifications imply the need for $Q$ values of 6 and 7 respectively or SNRs of 12 and 14 .

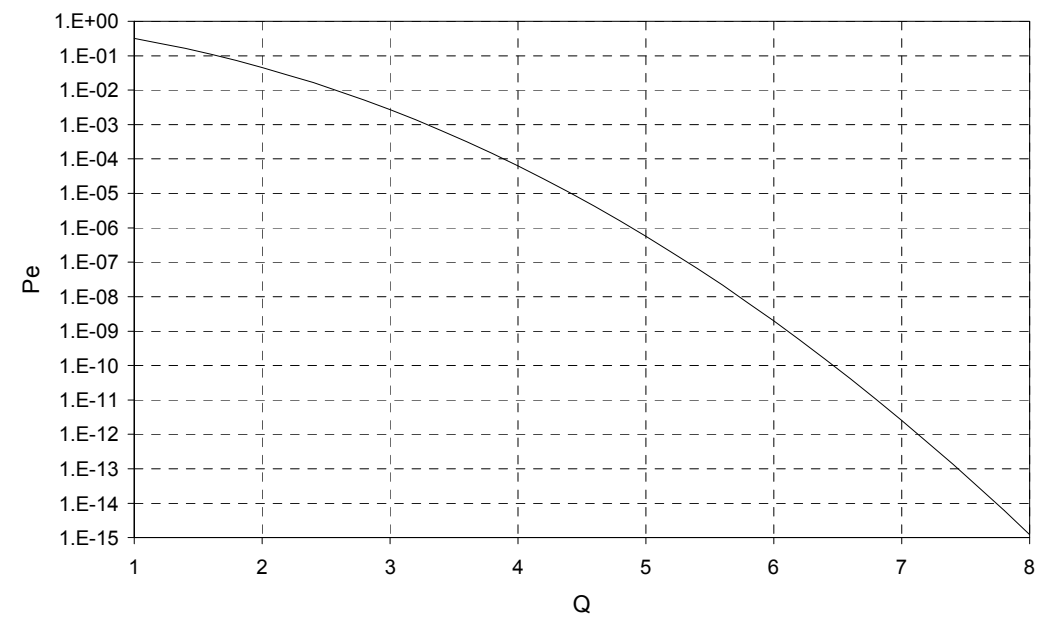

Figure 6: Error probability $\mathrm{P}_{\mathrm{e}}$ versus error probability factor $\mathrm{Q}$

In addition to the quantitative approach above, much qualitative information may be gained from generating what is known as the eye diagram. To observe statistical 
variations in the received signal we need to be able to display many bits (hundreds or even thousands) of both $1 \mathrm{~s}$ and $0 \mathrm{~s}$ in various sequences all superimposed on one another. This can be done by transmitting a square pulse non return to zero (NRZ) pseudo-random bit stream (PRBS) and displaying the receiver output on the oscilloscope which is triggered by the PRBS clock. In such an arrangement, the oscilloscope displays the signal every few clock cycles or indeed every clock cycle depending on the timebase setting. If we now utilise the persist function many signal sequences are displayed superimposed on each other and we can see the effects of the statistical phenomena, such as noise and jitter, on the signal features.

BER (COM) in conjunction with ED COM enables the generation and investigation of eye diagrams for various conditions of signal attenuation, dispersion and noise. In addition, it enables the measurements and recording of the 0 and 1 sampled signal levels for a large number of bit periods. From these the noise probability distribution functions and the rms noise voltage may be determined allowing the $Q$ factors and $B E R$ to be calculated.

Experimental Programme

The $B E R(C O M)$ educator kit enables the generation of eye diagrams for all the system permutations of OptoSci's Fibre Optic Communication educator kit (ED-COM). Under the experimental programme the students perform the following investigation:

- Generate and evaluate eye patterns and use them as a qualitative diagnostic tool

- Perform rise time, pulse width, jitter and noise measurements

- Examine bit-rate limitations due to noise, attenuation and dispersion

- Estimate Q-factors and Bit Error Rates (BER) from noise amplitude measurements on an oscilloscope

- Generate and analyse eye pattern histograms with OptoSci's Q-factor analysis software to estimate Q-factors and Bit Error Rates

- Examine and compare LED and Laser responses over different fibre lengths and at various bit rates

Some of the results from a typical set of measurements obtained using ED-COM and $\mathrm{BER}(\mathrm{COM})$ will be examined in more detail in the following section.

\section{Sample Results and Analysis}

\section{Typical Eye Diagram Measurements}

Figure 7 illustrates the typical measurements which are made on an eye diagram in order to provide semi-quantitative information about the transmission quality of a communications link. 

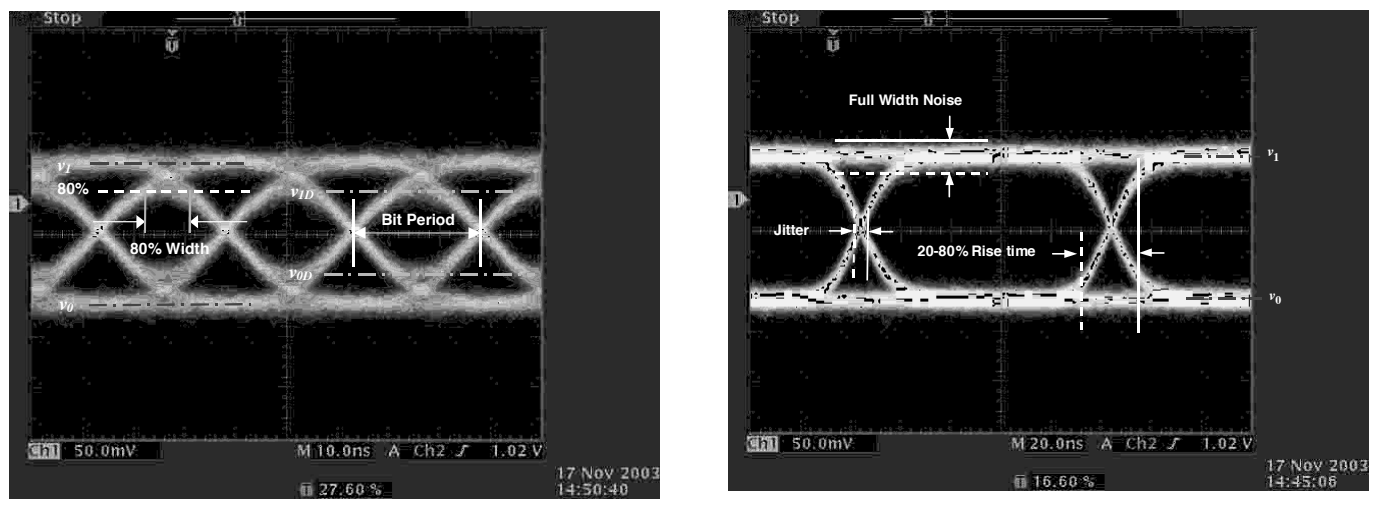

Figure 7: Sample measurements on eye diagrams.

\section{Investigation of Eye Diagrams for LED Transmitter}

The eye diagrams for LED transmission at 20 and $40 \mathrm{Mbit} / \mathrm{s}$ through each of $2 \mathrm{~km}, 3 \mathrm{~km}$ and $4 \mathrm{~km}$ of fibre are displayed in Figure 8.

As the transmission length increases at 20Mbit/s, the signal strength and SNR decrease due to signal attenuation. The effects of dispersion also become more evident with the rise time increasing with longer link lengths and the $80 \%$ pulse width decreasing as a percentage of the bit period. Pulse spreading with increasing rise times and decreasing width as a fraction of the bit period serves to increase the BER as the available sampling window in the centre of the pulse reduces. However, at 20Mbit/s the effects of dispersion on the BER are limited since the bit period is long and there is significant tolerance for the timing of the sampling point. The threshold level and the Q-factor decrease with length and of course this will be accompanied by an increase in the BER from this effect.

At $40 \mathrm{Mbit} / \mathrm{s}$ as the transmission length increases, the signal strength and SNR similarly decrease due to signal attenuation. However, the effects of dispersion now become very significant. The rise time increases to equal the bit period and the $80 \%$ pulse width reduces to a small percentage of the bit period. Coupled with increasing pulse jitter and jitter on the sampling clock there is an increasing probability that the sampling point will be off centre and not coincident with the peak of the signal, resulting in increased BER. In addition, for distances of $3 \mathrm{~km}$ and beyond, the effects of inter symbol interference (ISI) can be clearly seen. The pulses have spread significantly into adjacent bit periods resulting in a reduction of the peak signal strength and an increase in the noise level. These effects serve to take the noise closer to the threshold level, with concomitant degradation in the BER. 


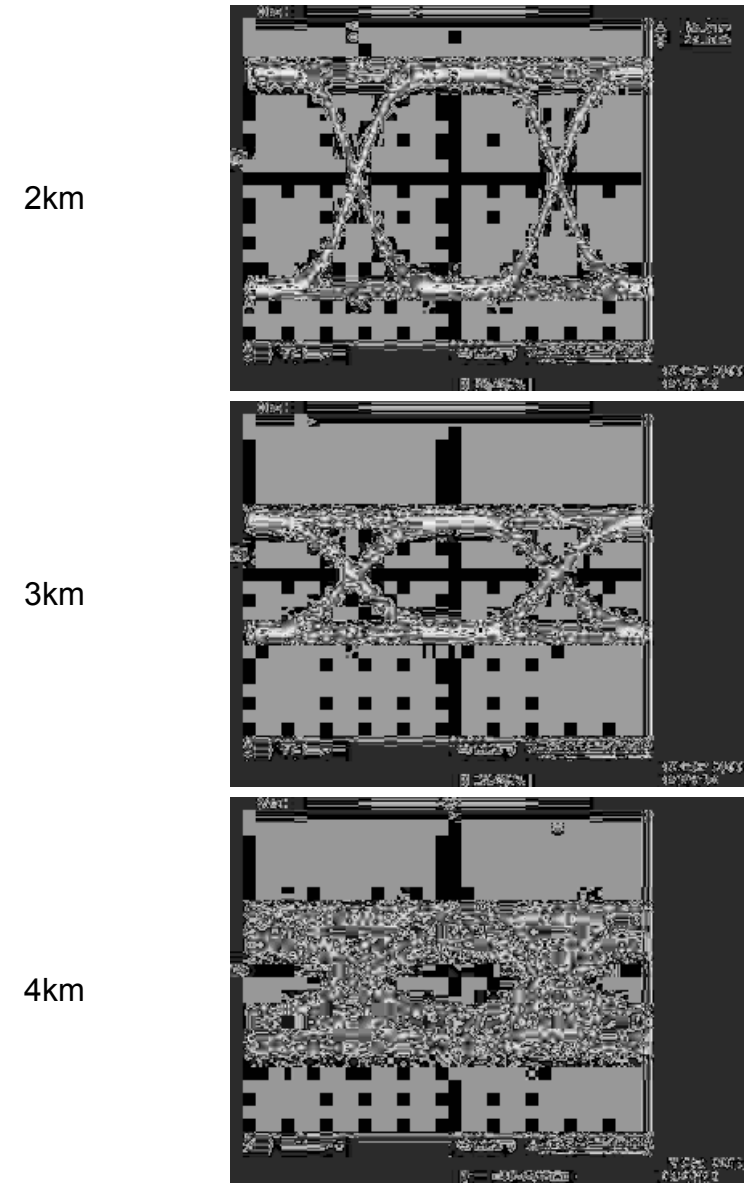

$20 \mathrm{Mbit} / \mathrm{s}$
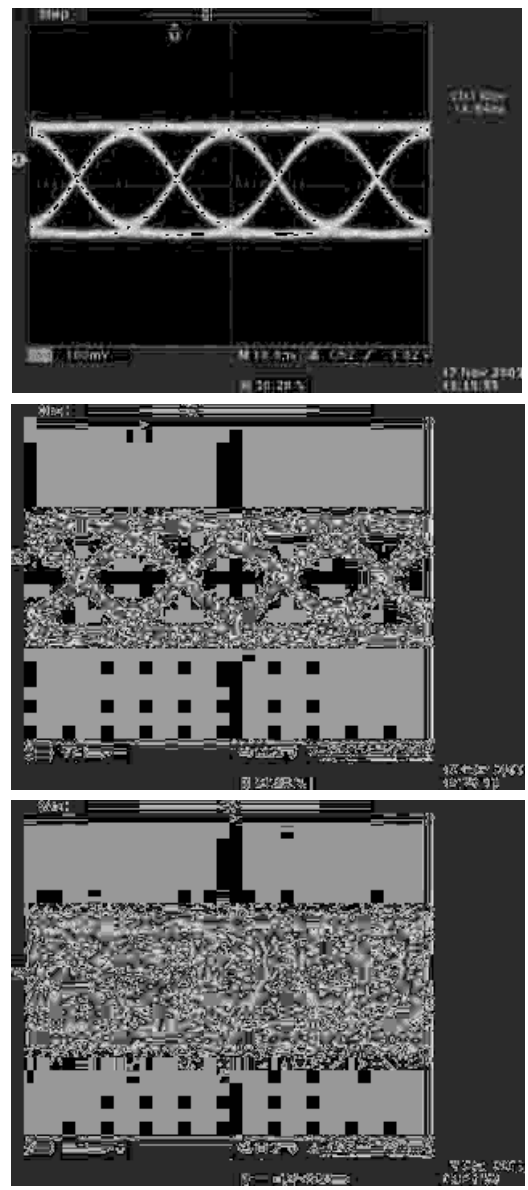

40Mbit/s

Figure 8: Eye diagrams for LED transmitter at 20 and $40 \mathrm{Mbit} / \mathrm{s}$ over $2 \mathrm{~km}, 3 \mathrm{~km}$, and $4 \mathrm{~km}$.

\section{Investigation of Eye Diagrams for Laser Transmitter}

Figure 9 depicts the eye diagrams for Laser transmission at 20 and $40 \mathrm{Mbit} / \mathrm{s}$ through each of $2 \mathrm{~km}, 3 \mathrm{~km}$ and $4 \mathrm{~km}$ of fibre.

The trends as regards SNR, Q-factor and BER are very similar to those for the LED except that the laser power at the input is considerably greater. The main differences arise from the effects of dispersion. The rise time and the $80 \%$ pulse width do not change as significantly with length for the laser relative to those for the LED. This is indicative of much lower dispersion. The LED linewidth is very broad $(>30 \mathrm{~nm})$ whilst the laser linewidth is narrow $(\sim 1 \mathrm{~nm})$. Hence, pulses from the LED experience a significant level of both material and inter-modal dispersion and spread much more quickly than the laser pulses for which material dispersion is insignificant due to the narrow laser 
linewidth. As a result of the reduced dispersion, it can be seen that the laser eye diagrams do not show any signs of significant ISI compared with the LED operating at $40 \mathrm{Mbit} / \mathrm{s}$ at distances over $3 \mathrm{~km}$.

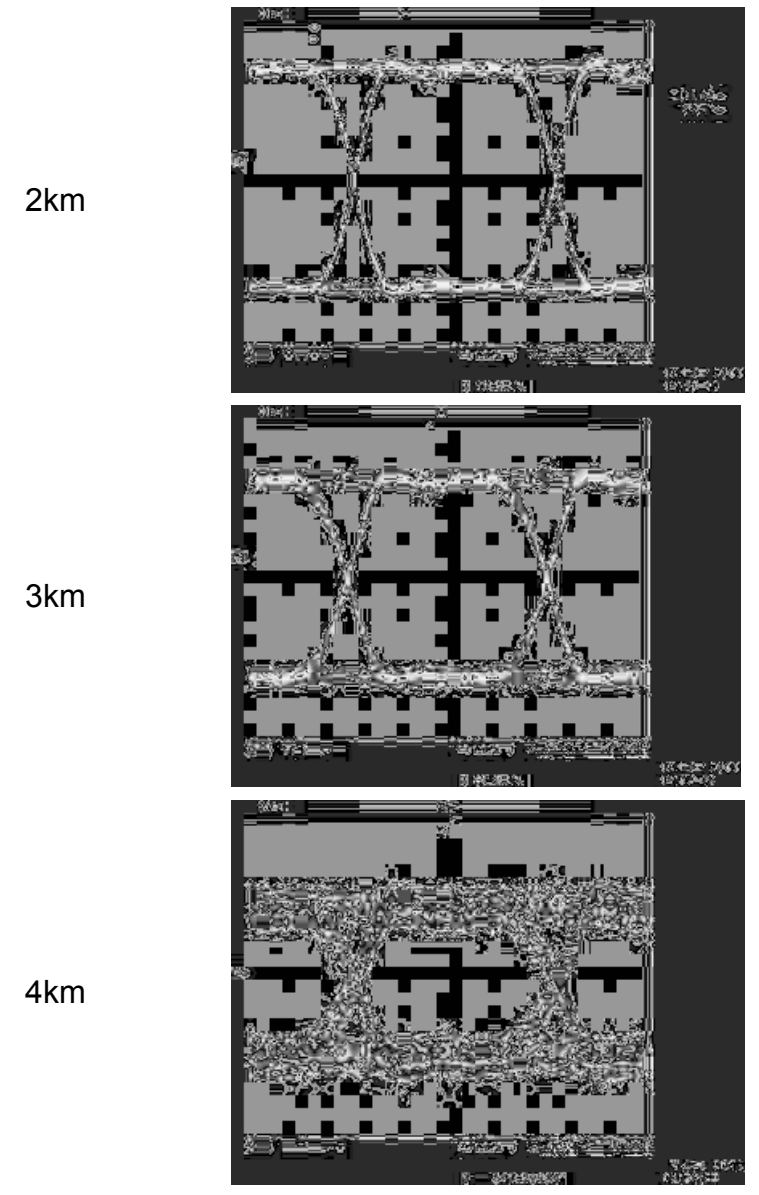

$20 \mathrm{Mbit} / \mathrm{s}$
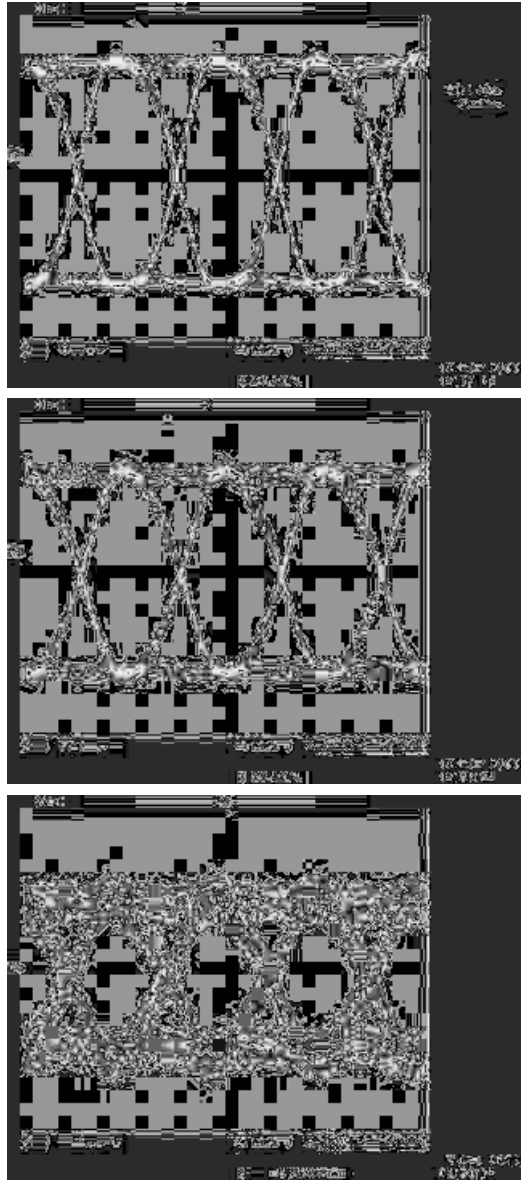

$40 \mathrm{Mbit} / \mathrm{s}$

Figure 9: Eye diagrams for Laser transmitter at 20 and $\mathbf{4 0} \mathrm{Mbit} / \mathrm{s}$ over $\mathbf{2 k m}, 3 \mathrm{~km}$, and $\mathbf{4 k m}$.

\section{Investigation of Q-factor and BER as a function of increasing link length with the} Q-factor Analysis Software

Using the Q-factor Analysis software described previously, the students establish the best fit Gaussian functions to the sampled data around the mean 0 and 1 signal levels in order to determine the Q-factor and the BER. Typical Q-factors and BER values for both the LED and laser transmitters operating at $40 \mathrm{Mb} / \mathrm{s}$ over $1-5 \mathrm{~km}$ are listed in Table 1. 


\begin{tabular}{|l||l|l||l|l|}
\hline \multicolumn{5}{|l|}{ Table 1: Q-factor \& BER variation with link length at 40Mb/s } \\
\hline \hline \multirow{4}{*}{ Length/km } & LED Transmitter & Laser Transmitter \\
\cline { 2 - 5 } & Q & BER & Q & BER \\
\hline 1 & 33.5 & $<10^{-25}$ & 21.6 & $<10^{-25}$ \\
\hline 2 & 11.2 & $<10^{-25}$ & 19.9 & $<10^{-25}$ \\
\hline 3 & 4.52 & $3.1 \times 10^{-6}$ & 21.2 & $<10^{-25}$ \\
\hline 4 & 2.43 & $7.6 \times 10^{-3}$ & 8.65 & $3.1 \times 10^{-18}$ \\
\hline 5 & 1.37 & $8.5 \times 10^{-2}$ & 3.15 & $8.2 \times 10^{-4}$ \\
\hline
\end{tabular}

High Q-factors in excess of about 10 and the corresponding BER values are pretty much meaningless essentially the BER is 0 in such situations. From Table 1 it can be seen that the Q-factor and BER both degrade as a function of increasing link length for both the LED and Laser transmitters, but those for the LED degrade much faster, particularly beyond $2 \mathrm{~km}$. Degradation occurs in both due to the attenuation in the fibre resulting in decreasing received signal power and SNR with increasing length. However, the degradation for the LED is more severe due to increased levels of pulse spreading and inter symbol interference. This is supported by the earlier risetime measurements, etc. and the evidence of ISI in the eye diagrams. There is no evidence of significant pulse spreading and ISI in risetime measurements or in the eye diagrams for the laser transmitter, so BER degradation for the laser arises mainly from attenuation. Pulses from the LED suffer greater spreading and ISI than those for the laser as a result of the increased linewidth of the LED output giving rise to a significant level of material dispersion in addition to intermodal dispersion. Material dispersion for the laser pulses is insignificant relative to intermodal dispersion due to the laser's narrow linewidth and pulse spreading does not significantly degrade the BER at lengths up to $5 \mathrm{~km}$.

\section{Investigation of Q-factor and BER as a function of increasing bit rate using the Q- factor Analysis Software}

The measured Q-factors and BER values for both the LED and laser transmitters operating over $4 \mathrm{~km}$ at bit rates of $10,20,30$ and $40 \mathrm{Mb} / \mathrm{s}$ are presented in Table 2.

\begin{tabular}{|c|c|c|c|c|}
\hline \multirow[b]{2}{*}{ Bit Rate/Mbs ${ }^{-1}$} & \multicolumn{2}{|c|}{ LED Transmitter } & \multicolumn{2}{|c|}{ Laser Transmitter } \\
\hline & Q & BER & $\bar{Q}$ & BER \\
\hline 10 & 6.86 & $3.4 \times 10^{-12}$ & 10.8 & $<<10^{-25}$ \\
\hline 20 & 6.29 & $1.6 \times 10^{-10}$ & 8.94 & $2.0 \times 10^{-19}$ \\
\hline 30 & 3.84 & $6.1 \times 10^{-5}$ & 8.83 & $5.2 \times 10^{-19}$ \\
\hline 40 & 2.43 & $7.6 \times 10^{-3}$ & 8.65 & $3.1 \times 10^{-18}$ \\
\hline
\end{tabular}

For a fixed link length the total fibre attenuation is fixed and degradation of the Q-factor and BER with increasing bit rate can only be caused by pulse spreading and ISI. Clearly from Table 2 there is significant degradation for the LED transmitter but little or no degradation for the laser. Both the laser and LED output pulses are affected similarly by intermodal dispersion, but only the LED output experiences significant levels of material dispersion due to its large linewidth. The additional contribution from material dispersion leads to the $Q$ and BER degradation seen for the LED transmitter, whereas the laser transmission, with only intermodal dispersion being significant, exhibits little degradation. 


\section{Conclusions}

In this paper we have described a laboratory educational package which has been developed to allow students to explore and examine the key components (sources, transmitters, fibre link and receiver) of an optical fibre communications system and experimentally investigate how the performance of such communication systems is limited by the effects of noise, attenuation and dispersion. This conceptual understanding of the performance of a fibre optic communications system is further developed in an extension module to the fibre optic communications package examining one of the primary figures of merit for a communications system, the bit error rate. This add-on module allows students to generate, evaluate and investigate signal quality trends using noise amplitude measurements on eye patterns and also statistical analysis of eye pattern histograms in order to appreciate the bit-rate limitations imposed on communication systems by noise, attenuation and dispersion.

\section{References}

1. See www.optosci.com for extensive additional information on OptoSci's range of photonics educator kits.

2. W. Johnstone, B. Culshaw, D. Moodie, I. Mauchline and D. Walsh, "Photonics laboratory teaching experiments for scientists and engineers" 7th international conference on Education and Training in Optics and Photonics (ETOP), Singapore 2001, and SPIE Proceedings 4588-51, 2002.

3. W. Johnstone, B. Culshaw, D. Walsh, D. Moodie and I. Mauchline, "Photonics laboratory experiments for modern technology based courses", IEEE Proceedings: Special issue on Electrical and Computer Engineering Education, pp41-54, 1999.

4. G. Keiser, Optical Fibre Communications, McGraw Hill, $3^{\text {rd }}$ Edition, 2000 